\title{
Supported microwires for electroanalysis: sensitive amperometric detection of reduced glutathione
}

Kamonwad Ngamchuea, Chuhong Lin, Christopher Batchelor-McAuley, Richard G. Compton*

* corresponding author: Richard G. Compton, Department of Chemistry, Physical \& Theoretical Chemistry Laboratory, University of Oxford, South Parks Road, Oxford, OX1 3QZ, United Kingdom

Email: richard.compton@chem.ox.ac.uk. Tel: +44(0)1865275957 Fax: +44(0)1865275410

\begin{abstract}
A carbon microfiber ( $7 \mu \mathrm{m}$ diameter) is employed herein as an electroanalytical sensor. The fabricated sensor is cheap, disposable and requires only $150 \mu \mathrm{L}$ of samples. The carbon fiber is surface-mounted onto an inert surface to overcome the problems of the fragility of the microwire and the possible interference of convective force due to the non-rigid nature of the wires, as well as to improve the reproducibility in length and the amperometric responses. As the cylindrical electrode is supported on a surface, the diffusion of redox-active species to the electrode is partially blocked by the substrate. A theoretical model is developed to account for this hindered diffusion. The mass-transport regime is altered from 'linear' at very short time, where the amperometric responses of the supported microwire closely resemble that of an isolated free standing cylinder (current $\propto$ electrode area), to 'convergent' at long time where its response now tends towards that of a hemi-cylinder of equal radius. The model is validated using chronoamperometry and cyclic voltammetry of an ideal outer-sphere redox probe, reversible ferrocene methanol oxidation. The fabricated microwire electrode is further applied to the system of irreversible 2-nitro-5-thiobenzoate oxidation used in the detection of reduced glutathione (GSH). The microwire electrode shows significantly higher ratio of Faradaic to non-Faradaic currents as compared to microdisk, macrodisk or carbon nanotube modified electrodes. Using the fabricated microwire, GSH can be detected with the sensitivity of $0.7 \mathrm{nA} \mu \mathrm{M}^{-1}$ and the limit of detection of $0.5 \mu \mathrm{M}(3 \mathrm{~s} / \mathrm{m})$.
\end{abstract}




\section{Introduction}

Providing rapid and potentially inexpensive analysis, electrochemical sensors have found extensive analytical application in areas ranging from environmental and industrial detectors to medical diagnosis. ${ }^{1-5}$ However, the limit of detection of such devices is often restricted by the presence of relatively large non-Faradaic (capacitative) currents associated with the charging of the electrode surface. ${ }^{6}$ In an attempt to resolve this problem, electrodes with microscopic $(\mu \mathrm{m})$ dimension have been employed to first enhance the mass-transport of the redox-active analyte to the electrode surface (altering the mass-transport regime from linear to convergent), and second to decrease the non-Faradaic contribution to the electrochemical response. Examples of such electrode designs include microdisk, ${ }^{7}$ microband electrodes, ${ }^{8,9}$ and microelectrode arrays. ${ }^{10-14}$ In comparison to more conventional macroelectrodes, these devices are relatively expensive, difficult to fabricate and can require calibration. Hence, such devices are not ideal for disposable use. More importantly, despite the theoretical expectations of the performance of these small electrodes, in practice they encounter difficulties relating to the presence of stray and/or parasitic capacitances arising from faults in the manufacturing process. The resulting capacitative currents are often found to be greater than that predicted on the basis of the geometric area of the electrode and hence limits the analytical sensitivity of the system. ${ }^{15-17}$

We herein present surface supported carbon fiber microwires as an alternative choice of electrode for analytical applications. These electrodes are shown to be accurate, reliable and appropriate for disposable use. Recent work in the literature has reported low limits of detection when using isolated microcylinder electrodes, for example in the detection of oxygen $(\sim 10 \mu \mathrm{M})^{17}$ and nanoparticles $(\sim 0.1 \mathrm{pM}) .{ }^{18,19}$ However the application of such electrodes has been limited by their fragility and issues in reproducibly producing electrodes of the same length. So far due to the use as isolated microcylinder these electrodes have been limited to being shorter than $1 \mathrm{~mm}$ in length as importantly, the use of long non-rigid electrodes moving freely in solution can give rise to a significant level of convective masstransport, affecting the accuracy of the analytical procedure. In this work to overcome this problem and enhance the durability of the microwire, the carbon fiber is surface mounted on a glass slide, alongside a conducting wire and a silver wire as counter and reference electrodes respectively forming a sensor platform (Fig. 1) which requires only a small sample volume (ca. $150 \mu \mathrm{L}$ ) for analysis. 
The supported microwires are used to quantify the concentration of a solution phase analyte via two electrochemical techniques: chronoamperometry and cyclic voltammetry. Quantitative analysis of the resulting voltammogram is made by the solving of the diffusion equation for the system's geometry subjected to appropriate boundary conditions. Although the theoretical response of an isolated (azimuthally symmetric) microcylinder electrode is well established, in the present case of supported microwires, diffusion to the electrode is partially blocked by the supporting surface. The amperometric response of this system differs from that of an isolated electrode, where for the latter case the current-time response (chronoamperometry) and the voltammetric peak currents (cyclic voltammetry) can be predicted on the basis of the work of Szabo et al. (eqn. 5) ${ }^{20}$ and Aoki et al. (eqn. 7) ${ }^{21}$ respectively. Consequently, herein a theoretical model is developed for the diffusion-only response of a surface supported microwire electrode. This model is validated experimentally using the ideal outer-sphere redox probe ferrocene methanol. ${ }^{22}$ Working curves of the peak currents predicted for cyclic voltammetry as a function of scan rates and for chronoamperoamperometry as a function of transient times are also presented for prospective future applications without the need for resource to further simulation.

This paper continues by demonstrating the analytical application of the newly developed electrochemical system. Specifically, the solution phase concentration of glutathione (GSH) is quantified electrochemically at micromolar concentrations ( $5 \mu \mathrm{M}$ to $65 \mu \mathrm{M}$ range, LOD $\left.0.5 \mu \mathrm{M}\left(3 \mathrm{~s}_{\mathrm{B}} / \mathrm{m}\right)\right)$, via its reaction with Ellman's reagent, 5,5'-dithiobis(2-nitrobenzoic acid) (DTNB). The irreversible reaction between DTNB and GSH results in the formation of 2nitro-5-thiobenzoate (TNB), the latter is electrochemically detectable at the carbon fibre electrode. GSH is an important anti-oxidant in humans and is present in biological samples at low micromolar concentrations ( $\sim 4 \mu \mathrm{M}$ in plasma, ${ }^{23}$ and $\sim 3 \mu \mathrm{M}$ in saliva $\left.{ }^{24}\right) .{ }^{25-27}$ At these low levels, measurements using macro- or micro-disk electrodes are demonstrated to be unrealizable.

\section{Experimental}

\subsection{Chemical reagents and instrumentation}

Reduced L-glutathione (GSH, $\geq 98.0 \%$ ), 5,5'-dithiobis(2-nitrobenzoic acid) (DTNB, $\geq 98 \%$ ), disodium ethylenediaminetetraacetate salt dihydrate (EDTA, 99.0-101.0\%), sodium 
phosphate dibasic $\left(\mathrm{Na}_{2} \mathrm{HPO}_{4}, \geq 99.0 \%\right)$ and sodium phosphate monobasic dihydrate $\left(\mathrm{NaH}_{2} \mathrm{PO}_{4} \cdot 2 \mathrm{H}_{2} \mathrm{O}, \geq 99.0 \%\right)$ were purchased from Sigma-Aldrich and were used as received without further purification. Solutions were prepared using deionized water (Millipore) with a resistivity of $18.2 \mathrm{M} \Omega \mathrm{cm}$.

Electrochemical experiments were performed in a thermostated $\left(25.0 \pm 0.2^{\circ} \mathrm{C}\right)$ Faraday cage, using a $\mu$ Autolab Type III potentiostat (Utrecht). All measurements were conducted using standard three electrode setups employing different working electrodes: glassy carbon $(\mathrm{CH}$ Instruments, $3 \mathrm{~mm}$ diameter), multi-walled carbon nanotube screen-printed (Dropsens, $4 \mathrm{~mm}$ diameter), micro carbon fiber microdisk (ALS, $7 \mu \mathrm{m}$ diameter) and the fabricated carbon fiber microwire electrodes (Section 2.2). Saturated calomel (BASi) or silver wire was used as a reference electrode; refer to SI (Table S1).

\subsection{Electrode design}

A carbon fiber, a silver wire and a metal conducting (tinned copper) wire were used as the working, reference and counter electrodes respectively. Carbon fiber ( $7 \mu \mathrm{m}$ diameter) was obtained from Goodfellow, UK. The carbon fiber was attached to a conducting wire $(0.2 \mathrm{~mm}$ diameter) of ca. $4 \mathrm{~cm}$ in length using the electrically conducting silver loaded epoxy adhesive (RS, USA) to allow the microfiber working electrode to be connected to electrochemical measurements. All electrodes were placed on a glass slide and sealed on both sides with the electrically insulating Kapton ${ }^{\circledR}$ tape to give the length of the electrodes of $1.0 \mathrm{~cm}$. Nail polish (nitrocellulose in a mixture of organic solvents) was used to form a hydrophobic barrier and make a well surrounding the electrodes. With the well size of $1.4 \times 1.4 \mathrm{~cm}^{2}$, a droplet of ca. $150 \mu \mathrm{L}$ of solution is required to cover the electrode and enable electrochemical measurement.

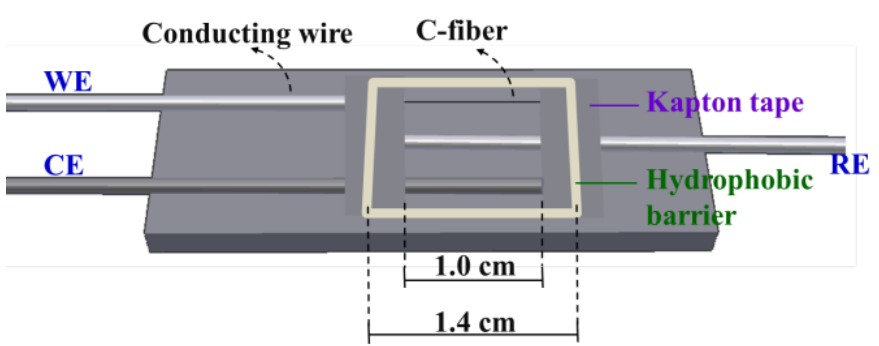

Figure 1: Design of the electrodes set-up on top of a non-conducting substrate with a C-fiber (7 $\mu$ m diameter), a Ag wire and a metal conducting wire as working, reference and counter electrodes respectively. 


\subsection{Characterization of the supported microwire electrodes: ferrocenemethanol $\left(\mathrm{FCCH}_{2} \mathrm{OH}\right)$ experiments}

The ferrocenemethanol/ferrocenium methanol $\left(\mathrm{FcCH}_{2} \mathrm{OH} / \mathrm{FcCH}_{2} \mathrm{OH}^{+}\right)$redox couple was used to characterize the fabricated wire electrodes. Cyclic voltammetry $(E=-0.2 \rightarrow 0.6 \rightarrow$ $0.2 \mathrm{~V}$ vs. SCE) of $1.0 \mathrm{mM} \mathrm{FCCH} \mathrm{mH}_{2} \mathrm{OH}$ the presence of $0.1 \mathrm{M}$ potassium chloride $(\mathrm{KCl})$ supporting electrolyte was performed at the carbon microfiber wire electrodes at varied scan rates $\left(25-400 \mathrm{mV} \mathrm{s}^{-1}\right)$. The position of the anodic peak potential of the voltammogram is noted and the overpotential is selected for chronoamperometric measurements. Hence for chronoamperometry, the potential of $0.35 \mathrm{~V}$ vs. SCE is applied and the transient currents recorded for $10 \mathrm{~s}$ at a sampling rate of $10 \mathrm{~K} \mathrm{~Hz}$.

\subsection{Application of the supported microwire electrodes: reduced glutathione (GSH) measurement}

The use of the surface supported microwire electrodes are demonstrated for the analysis of an irreversible system, 5-thio-2-nitrobenzoate (TNB) oxidation. This species is significant for the detection of reduced glutathione (GSH). GSH cannot be electrochemically detected directly in aqueous media. Solutions of GSH $(5-65 \mu \mathrm{M})$ were first reacted with excess 5,5'dithiobis(2-nitrobenzoic acid) (DTNB, $170 \mu \mathrm{M}$ ); see eqn. 1. The reaction produces 5-thio-2nitrobenzoate (TNB) which is detected electrochemically using the microwire electrodes.

$$
\mathrm{DTNB}+\mathrm{GSH} \longrightarrow \mathrm{GS}-\mathrm{TNB}+\mathrm{TNB}
$$

Note that the measurement of free thiol concentration requires the presence of a buffer for the thermodynamically-driven reaction of thiols with DTNB. ${ }^{28}$ Hence, the chemical reactions were performed in the phosphate buffer $\mathrm{pH} 7.5$ and allowed to mix for 2 min prior to the electrochemical measurements. ${ }^{29}$ The resulting reaction mixtures are then subjected to cyclic voltammetry $\left(E=0.2 \rightarrow 0.8 \rightarrow 0.2 \mathrm{~V}\right.$ vs. Ag, at the scan rate of $25 \mathrm{mV} \mathrm{s}^{-1}$ ) or chronoamperometry ( $E=0.75 \mathrm{~V}$ vs. Ag) at the glassy carbon, carbon nanotube screenprinted, carbon microdisk or supported microwire electrode. 


\section{Theory and simulation}

Numerical simulation is applied to understand the electrochemical behaviour of a microcylinder located flat and lengthened on an inert substrate. The geometry of the simulation model is shown in Figure 2 and the concentration of the reactant $c$ is calculated as a function of time $t$ in the Cartesian coordinate system $(x, y, z)$. The microclyinder is assumed long enough that only the circumference of the microcylinder electrode need to be considered in the simulation, reducing the problem to two-dimensions $(x, z)$. Assuming that the masstransport of the reactant in the solution is only due to diffusion and that a reversible oneelectron transfer reaction occurs at the surface of the supported microcylinder surface, the whole system can be described by the equations listed in Table 1. For a fast one-electron oxidation reaction, the concentration of the reactant on the electrode surface $c_{\text {surf }}$ can be calculated via the Nernst equation:

$$
c_{\text {surf }}=\frac{1}{1+\exp \left(\frac{F\left(E-E_{\mathrm{f}}\right)}{R T}\right)}
$$

where $F$ is the Faraday constant, $R$ is the gas constant and $T$ is the absolute temperature. $E$ is the applied potential on the electrode, $E_{\mathrm{f}}$ is the formal potential of a redox reaction, and the difference between $E$ and $E_{\mathrm{f}}$ is the overpotential for a redox reaction. The simulation grids are built in the same way as applied for a sphere on a plate, details of which can be found in our previous work. $^{30,31}$

In this work, two electrochemical techniques, chronoamperometry and cyclic voltammetry, are used to explore the electrochemical reaction at the microcylinder electrode on a flat plate. Chronoamperometry measures the current varying as a function of the experimental time at a fixed electrode potential. In this paper, all the chronoamperometry results are simulated at a high overpotential, where the surface concentration of the reactant is effectively zero. Cyclic voltammetry reflects the current response at a time varying electrode potentials. The potential $E$ is expressed by the following function of the experimental time $t$ and the scan rate $v$ : 


$$
E=\left\{\begin{array}{c}
E_{\mathrm{ini}}+v t, \quad t \leq \frac{\left|E_{\mathrm{rev}}-E_{\mathrm{ini}}\right|}{v} \\
E_{\mathrm{rev}}-v\left(t-\frac{E_{\mathrm{rev}}-E_{\mathrm{ini}}}{v}\right), \quad t>\frac{\left|E_{\mathrm{rev}}-E_{\mathrm{ini}}\right|}{v}
\end{array}\right.
$$

where $\left[E_{\text {ini }}, E_{\text {rev }}\right]$ defines the potential window of the cyclic voltammogram and $E_{\text {ini }}<E_{\text {rev }}$ for an oxidation process. For a microcylinder on a plate with the length of $l$, the current $I$ can be calculated from the gradient of the reactant concentration $c$ at the electrode surface:

$$
I=-F D \iint_{S} \frac{\partial c}{\partial n} d S
$$

where $n$ is the unit normal vector pointing away from the microcylinder surface and $S$ refers to the area of the microcylinder excluding the ends of the electrode.

The resulting problem was solved numerically by means of the Newton-Raphson method and the alternating direction implicit (ADI) method. ${ }^{32}$ The simulation programmes were written in Matlab R2016a and performed using an Intel(R) Xeon(R) 3.60G CPU.

\begin{tabular}{|c|c|}
\hline Condition & Equation \\
\hline$t=0$ & $\begin{array}{l}c c=c^{*} \\
\left(c^{*} \text { is the concentration in }\right. \\
\text { the bulk solution) }\end{array}$ \\
\hline$Z \rightarrow \infty$ & $c=c^{*}$ \\
\hline$x \rightarrow \infty$ & $c=c^{*}$ \\
\hline$z=0$ & $\frac{\partial c}{\partial z}=0$ \\
\hline $\begin{array}{c}x=0, z>2 r_{\mathrm{el}} \\
\left(r_{\mathrm{el}} \text { is the radius of the }\right. \\
\text { microcylinder })\end{array}$ & $\frac{\partial c}{\partial x}=0$ \\
\hline$\left(z-r_{\mathrm{el}}\right)^{2}+x^{2}=r_{\mathrm{el}}^{2}$ & $C=\frac{1}{1+\exp \left(\frac{F\left(E-E_{\mathrm{f}}\right)}{R T}\right)}$ \\
\hline$x, z$ in the solution & $\begin{array}{c}\frac{\partial c}{\partial t}=D\left(\frac{\partial^{2} c}{\partial x^{2}}+\frac{\partial^{2} c}{\partial z^{2}}\right) \\
\text { ( } D \text { is the diffusion } \\
\text { coefficient of the reactant) }\end{array}$ \\
\hline
\end{tabular}

Table1: Conditions and equations used in the simulation of the microcylinder on a flat plate system.
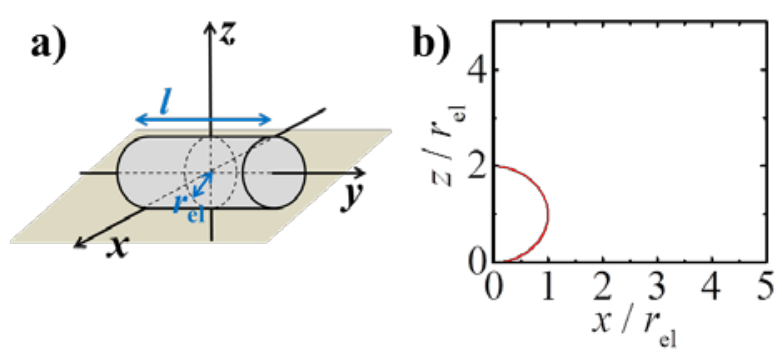

Figure 2: a) Geometry of the microcylinder electrode; b) Simulation model of the microcylinder electrode supported on the substrate. The red curve refers to the electroactive surface in the two-dimension $(x, z)$ coordinate. $r_{\mathrm{el}}$ is the radius of the microcylinder. 


\section{$4 \quad$ Results and discussions}

Two electrochemical techniques, chronoamperometry and cyclic voltammetry, are studied at the surface supported microwire electrodes. Both numerical simulation and experimental results are presented. First, the results of the simulation are detailed and compared to the responses of both a hemi and full (free standing) microcylinder. The simulation results are subsequently experimentally validated using a reversible one-electron transfer process $\left(\mathrm{FCCH}_{2} \mathrm{OH} / \mathrm{FCCH}_{2} \mathrm{OH}^{+}\right)$. Finally, the well-characterized surface supported microwire electrodes and cell design is then employed in the analysis of the more complicated system of irreversible TNB (from GSH + DTNB) oxidation.

\subsection{Chronoamperometry}

The chronoamperometric response of a fast one-electron transfer reaction at a surface mounted microwire electrode is explored via simulation and experiment. The effect of the wire geometry on the current-time responses is explored in relation to the response of an isolated free standing cylinder and a hemi-cylindrical electrode.

\section{Theory}

The predicted chronoamperometry of the fast one-electron transfer reaction at high overpotential on the surface supported microwire electrode is shown in Figure 3. For clarity, the axes in Figure 3 are normalized current $I /\left(2 F l D c^{*}\right)$ and normalized time $D t / r_{\mathrm{el}}{ }^{2}$. Figure 3 contains the chronoamperograms on the supported microwire electrode (black line), the isolated free standing full cylinder microwire (blue line) and the hemi-microcylinder (red line). The radii of the three microwire electrodes are all the same. The chronoamperogram of the supported microwire is obtained from the simualtion. The current-time responses of the another two geometries have been analytically approximated in the literature ${ }^{20}$ :

$$
\frac{i(t)}{2 \pi \ln e_{e} F D c^{*}}=\sigma_{\mathrm{geo}}\left(\frac{e^{-\sqrt{\pi \tau} / 10}}{\sqrt{\pi \tau}}+\frac{1}{\ln \left(\left(4 \tau e^{-\gamma}\right)^{0.5}+e^{5 / 3}\right)}\right), \tau=\frac{D t}{r_{\mathrm{el}}^{2}}
$$

where $n_{\mathrm{e}}$ is the number of electrons transferred in the redox reaction ( $n_{\mathrm{e}}=1$ for the oneelectron-transfer reaction), $\gamma$ is approximated to be 0.5772156 , and $\sigma_{\text {geo }}$ reflects the geometry area of the microwire being 0.5 for a hemicylinder and 1 for a full cylinder. 


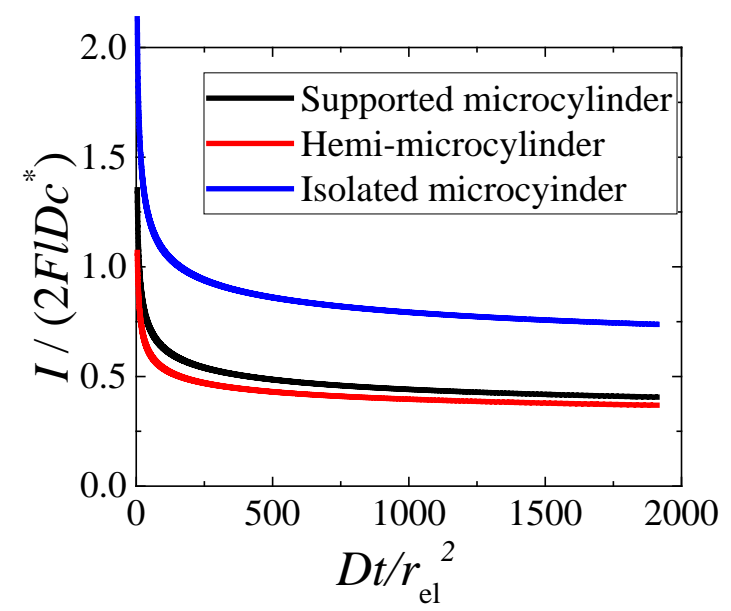

Figure 3 Chronoamperograms of a fast one-electron transfer reaction at high overpotential on microcylinder electrodes with various geometries. The black, red and blue solid lines are the simulation for the supported microwire, the hemi-microcylinder, and the isolated microwire respectively.

As shown in Figure 3, the current-time responses for the one-electron transfer reaction are distinguished by the geometry of the microcylinder. The reaction current of the supported microcylinder is between the currents expected from the hemi-microcylinder and the isolated microcylinder. At very short times, the current is proportional to the surface area of the electrode, hence the response of the surface mounted microcylinder tends towards that of the fully isolated microwire. However, as time progresses and the thickness of the diffusion layer increases relative to the electrode radius, the importance of the diffusional blocking by the electrode support becomes a more signifcant factor. Consequently, the current at the surface supported microwire electrode tends towards the reponse predicted for the hemi-cylindral electrode. However, even in the long time limit as the currents become quasi-steady state, due to the differing gemotries associated with the surface supported microwire and the hemicylindral electrode the currents predicted for the two systems do not converge to the same value. Table 2 presents the ratios of chronoamperometric currents of a fast one-electron transfer reaction at the different electrode geometries in the long time limit $\left(D t / r_{\mathrm{el}}{ }^{2}>>1\right)$, demonstrating that the geometric influence cannot be neglected when analyzing electrochemical reactions at a microcylinder electrode. 
Table 2: Geometry influence on the predicted quasi-steady state currents of the different microwire electrode geometries. Values respresent the long-time limit current ratios among the supported microwire, the isolated microwire and the hemi-microcylinder with equal radii.

\begin{tabular}{|c|c|}
\hline Geometry & Current ratio at long time \\
\hline$i_{\text {supported }} / i_{\text {isolated }}$ & 0.545 \\
\hline$i_{\text {supported }} / i_{\text {hemi }}$ & 1.09 \\
\hline$i_{\text {hemi }} / i_{\text {isolated }}$ & 0.5 \\
\hline
\end{tabular}

\section{Experimental}

The reversible one-electron transfer redox couple of $\mathrm{FcCH}_{2} \mathrm{OH} / \mathrm{FcCH}_{2} \mathrm{OH}^{+}$is used to demonstrate the current-time responses at the supported microwire electrode. Experimentally, $\mathrm{FCCH}_{2} \mathrm{OH}$ of $1.0 \mathrm{mM}$ concentration (in $0.10 \mathrm{M} \mathrm{KCl}$ ) is oxidized at a potential of $0.35 \mathrm{~V}$ vs. SCE for $10 \mathrm{~s}$. The chronoamperograms obtained are then compared with the results of numerical simulation from the previous section. Due to the influence of non-faradaic contributions to the current response (see SI section S2.1.2), the data obtained during the course of the chronoamperogram before 0.3 seconds, after application of the electrode potential, are removed from the data set during analysis. The quality of the fit between the experimental and simulated data is assessed based on the magnitude of the mean scaled absolute deviation (\%MSAD) between the data sets. The \%MSAD is defined as:

$$
\% \operatorname{MSAD}=\frac{1}{N} \sum_{N}\left|\frac{I_{\text {sim }}-I_{\text {exp }}}{I_{\exp }}\right| \times 100
$$

Due to the alteration in the diffusion regime during the course of the chronoamperogram, it is important to analyze the optimal time range for the experimental analysis. ${ }^{33}$ The experimental time range of $0.3 \mathrm{~s}-3.8 \mathrm{~s}$ was found to give the lowest value of \%MSAD (0.31\%, see SI Fig. S3a). This time range is subsequently chosen for use in the following analysis.

In accordance with eqn. 5, at high overpotentials the current-time responses of a reversible one-electron transfer process at a microcylinder electrode varies as a function of diffusion coefficient $(D)$, concentration $\left(c^{*}\right)$ of the redox-active species, the radius $\left(r_{\mathrm{el}}\right)$ and length $(l)$ of the wire electrode. In present experimental case, the concentration of ferrocencemethanol and the radius of the electrode are precisely known to be $1.02 \mathrm{mM}$ and $3.5 \mu \mathrm{m}$ respectively.* 
* The carbon fiber wire has its diameter as specified by the manufacturer (Goodfellow, UK) of $0.007 \mathrm{~mm}$ with the tolerances in the fiber diameter, number of strands, tex number and length of $\pm 25 \%, \pm 10 \%, \pm 10 \%$ and $+5 \%$ / $-1 \%$ respectively.

There are however two unknown parameters, $D$ and $l$, noting that the length $l$ will vary slightly between electrode preparations (the question of the reproducibility of the wire length will be returned to later in the text). Consequently, a best fit of the experimental data needs to be found using these two parameters, this best fit is found by searching for a local minimum in the \%MSAD (see SI Section 2.1.2). The values of $D$ and $l$ which yield the lowest \%MSAD of $0.31 \%$ are $7.81 \times 10^{-10} \mathrm{~m}^{2} \mathrm{~s}^{-1}$ and $1.034 \mathrm{~cm}$ respectively; see SI Fig. S3b. The value of $D$ obtained is in excellent accordance to that reported by Sun and Mirkin, ${ }^{34}$ and Miao et al. ${ }^{22}$ $\left(7.8 \times 10^{-10} \mathrm{~m}^{2} \mathrm{~s}^{-1}\right)$.

Having validated the simulated chronoamperometric response of the surface mounted microwire electrode and utilized this as a method to accurately determine the diffusion coefficient of the redox species ferrocenemethanol, the work now continues by looking at the voltammetric response of these surface mounted microwire electrodes.

\subsection{Cyclic voltammetry}

Simulation of the cyclic voltammetry for a fast one-electron transfer process is first performed, providing a working curve for the predicted peak currents at a supported microwire electrode for a reversible one-electron diffusion only redox process. Experimentally, the $\mathrm{FCCH}_{2} \mathrm{OH} / \mathrm{FCCH}_{2} \mathrm{OH}^{+}$redox reaction is taken as the model system to study the cyclic voltammetric behaviour of the supported microwire electrode, and the results from simulation and experiments compared towards the end of this section.

\section{Theory}

Figure 4a shows the current-potential responses for a fast one-electron transfer reaction simulated under different scan rates and Figure $4 \mathrm{~b}$ compares the forward peak currents between the surface supported and isolated microwire electrodes. The peak current of the supported microwire are obtained from simulation. For the isolated microwire, the peak currents can be predicted by the following equation: ${ }^{21}$

$$
I_{\mathrm{p}}=2 \pi n_{\mathrm{e}} F c^{*} D l\left(0.446 p+0.335 p^{0.15}\right), p=\left(\frac{n_{\mathrm{e}} F v r_{\mathrm{el}}^{2}}{R T D}\right)^{0.5}
$$


As shown in Figure 4b, the ratios of the peak currents between the supported and the isolated

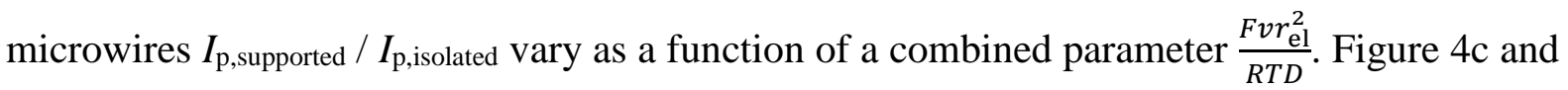
4d provide two example concentration profiles collected at the forward peak potentials with $\log _{10}\left(\frac{F v r_{\mathrm{el}}^{2}}{R T D}\right)=3.5$ and $\log _{10}\left(\frac{F v r_{\mathrm{el}}^{2}}{R T D}\right)=-4$, respectively. The combined parameter reflects the diffusion mode of the reactant. When the combined parameter is large, linear diffusion is observed as illustrated in Figure 4c, hence the current is proportional to the electrode surface area and thus the ratio $I_{\mathrm{p}, \text { supported }} / I_{\mathrm{p} \text {,isolated }}$ tends to unity at large values of $\frac{F v r_{\mathrm{el}}^{2}}{R T D}$. Conversely, when the value of the combined parameter decreases, the diffusion varies from linear to radial, as shown in Figure 4d. Diffusion to the bottom of the supported microcylinder is hindered due to the substrate and the geometry influence bemores more significant. At very

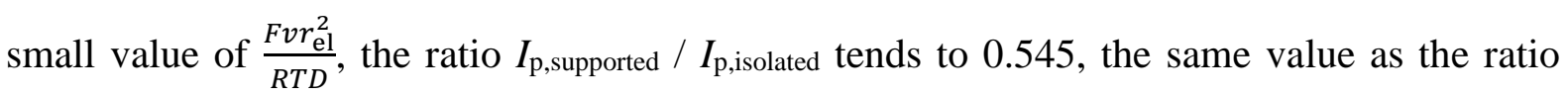
between two chronoamperometric currents listed in Table 2 . The exact values of the $I_{\mathrm{p} \text {,supported }}$

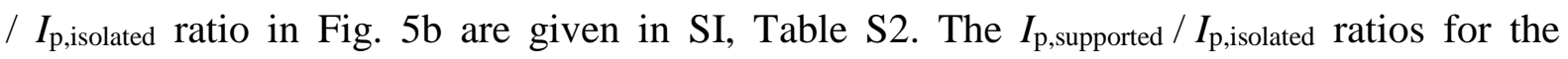
irreversible electron-transfer system are further provided in Section S2.2.2 in the SI.
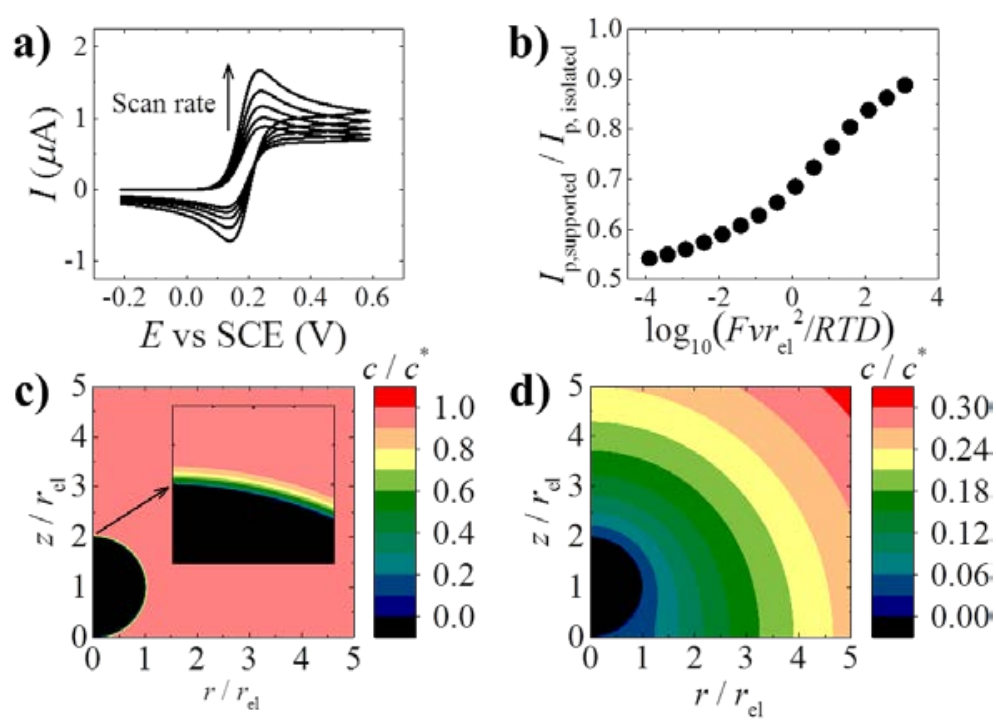

Figure 4: a) Cyclic voltammetry of a fast one-electron transfer reaction on the supported microwire electrode at different scan rates. From bottom to top, the scan rates are 25, 50, 100, 200 and $400 \mathrm{mV} \mathrm{s}^{-1}$; b) the ratio of forward peak currents between the supported and isolated microwire electrodes, see SI Table S2 for exact values; c) concentration profile of the reactant collected at the forward peak potential when $\log _{10}\left(F v r_{\mathrm{el}}^{2} / R T D\right)=$ 3.5 ; d) concentration profile of the reactant collected at the forward peak potential when $\log _{10}\left(F v r_{\mathrm{el}}^{2} / R T D\right)=-4$. 


\section{Experimental}

Cyclic voltammetry of ferrocenemethanol $\left(\mathrm{FcCH}_{2} \mathrm{OH}\right)$ of $1.0 \mathrm{mM}$ concentration (in $0.10 \mathrm{M}$ $\mathrm{KCl})$ was measured $(E=-0.2 \rightarrow 0.6 \rightarrow-0.2 \mathrm{~V}$ vs. Ag) at range of scan rates $(25-400 \mathrm{mV}$ $\mathrm{s}^{-1}$ ). The previously determined values of diffusion coefficient $(D)$ and length of wire electrode (l) of $7.81 \times 10^{-10} \mathrm{~m}^{2} \mathrm{~s}^{-1}$ and $1.034 \mathrm{~cm}$ respectively are used to analyze the voltammetric currents; refer to eqn. 7. As shown in Fig. 5a, the experimental results show an excellent fit to the numerical data, with the plots of comparison of peak potentials and peak currents provided in Fig. 5b. The results indicate that not only the quasi-steady state currents, but the fabricated microwire electrodes give well-defined CVs for a reversible redox system, and that the working curve presented in Fig. $4 \mathrm{~b}$ together with the analytical expression given in eqn. 7 can be used to accurately predict the voltammetric peak currents of the supported microwire electrodes.
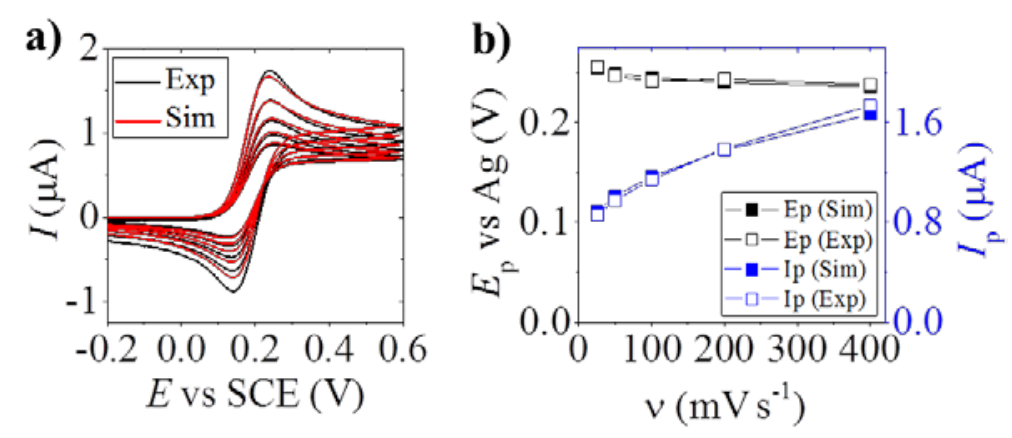

Figure 5: a) Comparison between the experimental (black) and simulated (red) cyclic voltammograms of $1.0 \mathrm{mM} \mathrm{FCCH}_{2} \mathrm{OH}(+0.10 \mathrm{M} \mathrm{KCl})$ oxidation, $v=25-400 \mathrm{mV} \mathrm{s}^{-1}, 298 \mathrm{~K}$ at the supported microcylinder electrode; b) comparison between the experimental (open squares, $\square$ ) and simulated (solid squares, $\square$ ) anodic peak potentials $\left(E_{\mathrm{p}}\right.$, black) and anodic peak currents ( $I_{\mathrm{p}}$, blue)

\subsection{Reproducibility of electrodes}

The above results have been presented for a supported microwire electrode. An important question arises as to the reproducibility of this cell design. Specifically, the variability in the electrode lengths between fabrications is of distinct importance. In the case that the electrochemical response of the wire is found to be highly reproducible, then within a certain error the electrode may be used without prior calibration of the wire length. The $1 \mathrm{~cm}$ carbon fiber wire electrodes were made by taping the Kapton tape on both sides of the carbon fiber. The error in the wire length arises due to the inherent variability of this process. The 
reproducibility of the electrode length and its resulting amperometric responses was tested using the well-characterized $\mathrm{FCCH}_{2} \mathrm{OH}$ as a model system.

Cyclic voltammetry of $1.0 \mathrm{mM} \mathrm{FCCH}_{2} \mathrm{OH}$ (in $0.1 \mathrm{M} \mathrm{KCl}$ ) at the scan rate of $25 \mathrm{mV} \mathrm{s}^{-1}$ was recorded on three different electrodes with three scans each. The oxidative peak potential remained at $0.246 \mathrm{~V}$ vs. SCE in all cases. The forward oxidative peak currents were $0.840 \pm 0.005 \mu \mathrm{A}$ within the electrode and $0.846 \pm 0.039 \mu \mathrm{A}$ between electrodes, giving the measurement errors of $0.6 \%$ and $4.6 \%$ within the electrode and between electrodes respectively. The length of the wires was calculated using eqn. 7 and the geometry factor ( $I_{\mathrm{p} \text {,supported }} / I_{\mathrm{p}, \text { isolated }}$ ) of 0.593 (Fig. $5 \mathrm{~b}$ ) to be $1.004 \pm 0.047 \mathrm{~cm}$, showing the excellent reproducibility of the electrodes; see SI Fig. S6 for raw data.

\subsection{Applications of the supported wire electrodes: GSH measurement}

Next, the supported microwire electrodes can be applied to a more complicated system such as that of an electrochemically irreversible process. The system chosen for investigation in this paper is 5-thio-2-nitrobenzoate (TNB). This compound is commonly used in the analysis of thiol concentrations, most notably reduced glutathione (GSH); see eqn. 1. TNB oxidation is an irreversible process and is thought to follow a CEC mechanism. ${ }^{35}$ Since GSH is present in biological samples at low micromolar levels ( $\sim 4 \mathrm{M}$ in plasma, ${ }^{23}$ and $\sim 3 \mu \mathrm{M}$ in saliva ${ }^{24}$ ), TNB produced from the reaction between GSH and DTNB is thus also at low concentration. Below, we will show that by simple linear sweep voltammetry, macrodisk electrodes, carbon nanotube modified electrodes and even microdisk electrodes cannot be used to detect GSH at this low micromolar level. On the other hand, the microwire electrodes developed herein display a limit of detection much lower than the biological relevant levels. Importantly, due to the disposable nature of the designed electrodes, they can be easily adopted for practical applications. The calibration curves of the quasi-steady state currents of the chronoamperograms and the peak currents of the cyclic voltammograms are presented herein as functions of GSH, and hence TNB concentrations.

\section{Enhanced sensitivity}

Four different carbon electrodes, glassy carbon macrodisk (GC), carbon nanotube modified screen-printed (CNT-SPE), carbon microdisk (C-microdisk) and supported carbon fiber microwire (C-wire), are next investigated for their sensitivity towards the voltammetric 
measurement of TNB oxidation. Figure 6 displays the voltammograms of the oxidation of $168 \mu \mathrm{M}$ TNB in $0.10 \mathrm{M}$ phosphate buffer $\mathrm{pH} 7.5$ at the scan rate of $25 \mathrm{mV} \mathrm{s}^{-1}$, currents are normalized relative to the electrode geometric area. The ratios of Faradaic peak current $\left(i_{\mathrm{F}}\right)$ to non-Faradaic current ( $i_{C}$, measurement of which is detailed in SI section S2.4.2) are observed to be $7.6 \pm 0.2,3.6 \pm 0.1,3.1 \pm 0.2$ and $33 \pm 4$ for GC (Fig. 6a), CNT-SPE (Fig. 6b), Cmicrodisk (Fig. 6c) and C-microwire (Fig. 6d) respectively.

It is predicted that Faradaic peak currents of the macrodisk electrodes vary linearly with the electrode area $\left(r_{\mathrm{el}}{ }^{2}\right)$, ${ }^{6}$ while that of the microdisk or microwire is dependent on the electrode radius $\left(\sim r_{\mathrm{el}}\right){ }^{21}$ Capacitance, on the other hand in the absence of considering other contributing factors, is linearly dependent on the area for all types of electrodes. ${ }^{6,36}$ Based on this knowledge, microdisks and microwires are expected to show better sensitivity than the macroelectrodes. The measurement at the microwire indeed shows significantly higher sensitivity (i.e. larger $i_{\mathrm{F}} / i_{\mathrm{C}}$ ) than the macroelectrodes, justifiable by the enhancement of the diffusion to the electrode and the reduction of the capacitance. The commercial microdisk electrodes however have unexpectedly large capacitance, likely reflecting the imperfect sealing of the electrodes and the occurrence of cracks potentially from the manufacturing processes. ${ }^{15-17}$ Note that the cyclic voltammogram provided by the supplier presented in Fig. S8 in the SI also shows similar level of capacitance. Accordingly, it has been demonstrated herein the superiority of the supported microwires for the analysis of low concentration of analyte where the measurement is often found to be challenging due to the large capacitance.
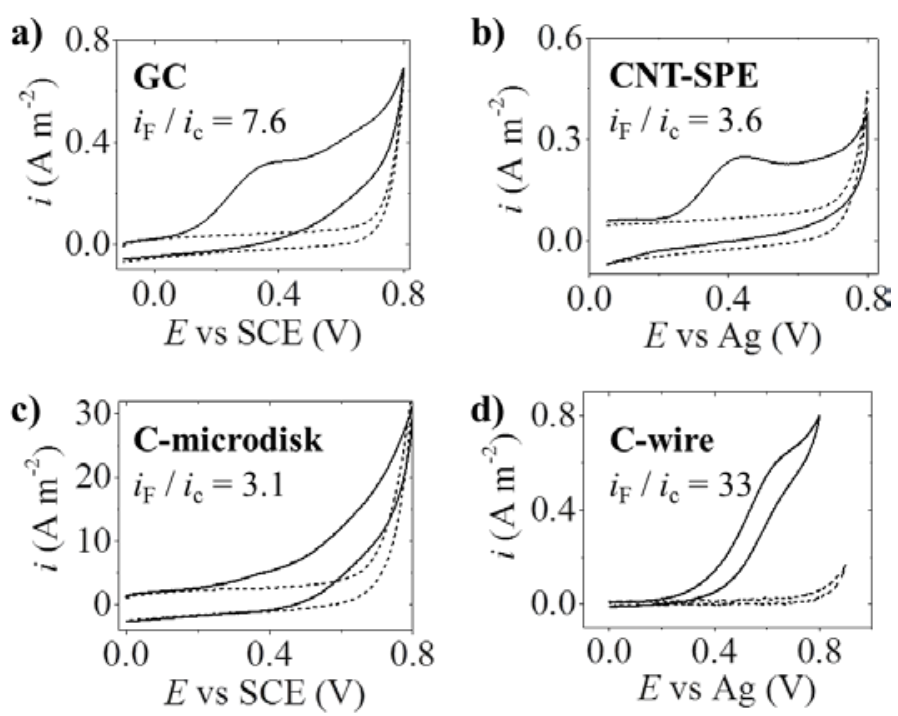

Figure 6: Cyclic voltammetry of $168 \mu \mathrm{M}$ TNB oxidation in $0.10 \mathrm{M}$ phosphate buffer $\mathrm{pH} 7.5$ at the scan rate of $25 \mathrm{mV} \mathrm{s}^{-1}$ at different carbon electrodes: a) glassy carbon macrodisk (GC); b) carbon nanotube modified screenprinted (CNT-SPE); c) carbon microdisk (C-microdisk); d) supported carbon fiber microwire (C-wire). The currents are normalized by the electrode area. 


\section{Calibration curve}

Having demonstrated the enhanced voltammetric response of the microwires, next the ability of the microwires to quantitatively detect GSH is explored.

An excess amount of DTNB $(168 \mu \mathrm{M})$ was used to react with $0,5,10,20,30,45$ and $65 \mu \mathrm{M}$ of GSH in $0.10 \mathrm{M}$, pH 7.5 phosphate buffer in the presence of $10 \mathrm{mM}$ ethylenediaminetetraacetic acid (EDTA; to prevent GSH oxidation catalyzed by metal ions impurity in the solutions ${ }^{29}$ ). The mixtures were left to react for 2 minutes to allow the reactions to go to completion, and hence producing $0,5,10,20,30,45$ and $65 \mu \mathrm{M}$ of TNB. Cyclic voltammetry of the reaction mixtures were recorded and the peak currents $[I(0.7 \mathrm{~V})-$ $I(0.3 \mathrm{~V})$ ] plotted against GSH concentrations, yielding a linear calibration plot $\left(\mathrm{R}^{2}>0.99\right)$ with the sensitivity of $0.7 \mathrm{nA} \mu \mathrm{M}^{-1}$ and limit of detection of $0.5 \mu \mathrm{M}\left(3 \mathrm{~s}_{\mathrm{B}} / \mathrm{m}\right)$; refer to Fig. 7 . Figure 7 further shows that other than TNB, no other species in the solution gives rise to the voltammetric response in the potential window studied.

Chronoamperometry was also recorded and the calibration plot of the quasi-steady state current at $10 \mathrm{~s}$ against GSH concentration gives the sensitivity of $0.7 \mathrm{nA} \mu \mathrm{M}^{-1}$ and the limit of detection of $1.3 \mu \mathrm{M}\left(3 \mathrm{~s}_{\mathrm{B}} / \mathrm{m}\right)$; refer to SI section 2.4.3.

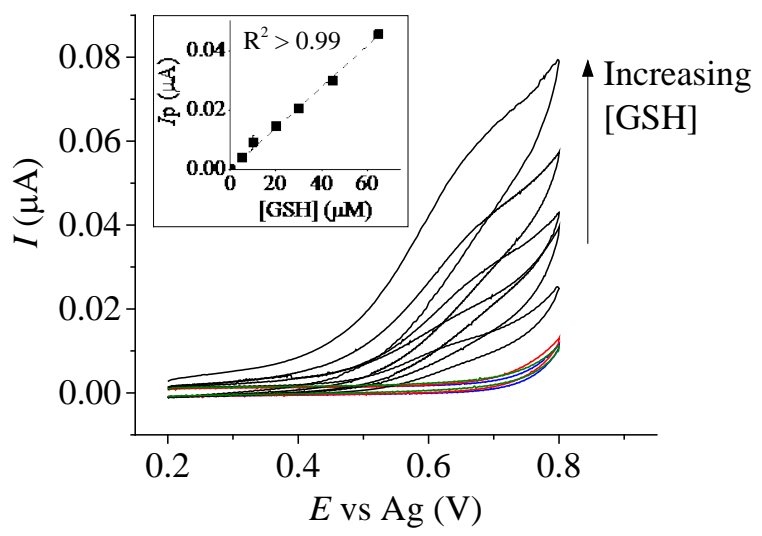

Figure 7: (Black) Cyclic voltammetry of $0,5,10,20,30,45$ and $65 \mu \mathrm{M}$ of GSH reacted with $168 \mu \mathrm{M}$ DTNB for 2 min to form equal concentrations of TNB at the supported microwire electrode. (Blue) Blank phosphate electrolyte. (Green) $60 \mu \mathrm{M}$ GSH (Red) $168 \mu \mathrm{M}$ DTNB. $v=25 \mathrm{mV} \mathrm{s}^{-1} .298 \mathrm{~K}$. Inlay: calibration plot of voltammetric peak currents $\left(I_{\mathrm{p}}\right)$ against GSH concentration. 


\section{Conclusions}

The fabricated supported microwires have been developed into an electrochemical sensor for the quantification of solution phase analytes at low micromolar concentrations. This sensor platform is reproducible, inexpensive and easily fabricated in-house without any requirement for specialized tools. The sensor requires only a small volume of samples ( $150 \mu \mathrm{L})$ and show good reproducibility in electrochemical responses $( \pm 4.6 \%)$. It displays excellent performance with the systems of reversible $\mathrm{FCCH}_{2} \mathrm{OH}$ oxidation and irreversible TNB oxidation studied in work, with sub-micromolar limit of detection. The theoretical models for the amperometric responses at the supported microwires are also developed for the first time in this work, reflecting the partially hindered diffusion of the analyte to the electrode blocked by the support.

\section{Acknowledgements}

$\mathrm{KN}$ is supported by DPST scholarship from the Royal Thai government. KN, CL, CBM and RGC acknowledge funding from the European Research Council under the European Union’s Seventh Framework Programme (FP/2007-2013)/ERC Grant Agreement no. [320403].

\section{References}

(1) Privett, B. J.; Shin, J. H.; Schoenfisch, M. H. Anal. Chem. 2008, 80, 4499-4517.

(2) Hanrahan, G.; Patil, D. G.; Wang, J. J. Environ. Monit. 2004, 6, 657-664.

(3) Toghill, K. E.; Compton, R. G. Int. J. Electrochem. Sc. 2010, 5, 1246-1301.

(4) Harfield, J. C.; Batchelor-McAuley, C.; Compton, R. G. The Analyst 2012, 137, 2285.

(5) Windmiller, J. R.; Wang, J. Electroanalysis 2013, 25, 29-46.

(6) Compton, R. G.; Banks, C. E. Understanding Voltammetry, 2nd ed.; Imperial College Press, 2011, p 444.

(7) Bond, A. M. The Analyst 1994, 119, 1R.

(8) Craston, D. H.; Jones, C. P.; Williams, D. E.; El Murr, N. Talanta 1991, 38, 17-26.

(9) Xiong, L.; Goodrich, P.; Hardacre, C.; Compton, R. G. Sens. Actuators, B 2013, 188, 978-987.

(10) Sokolov, S. V.; Bartlett, T. R.; Fair, P.; Fletcher, S.; Compton, R. G. Anal. Chem. 2016, 88, 8908-8912.

(11) Rahman, A. R.; Guiseppi-Elie, A. Biomed. Microdevices 2009, 11, 701-710.

(12) Prieto, F.; Coles, B. A.; Compton, R. G. J. Phys. Chem. B 1998, 102, 7442-7447.

(13) Streeter, I.; Compton, R. G. J. Phys. Chem. C 2007, 111, 15053-15058.

(14) Ordeig, O.; del Campo, J.; Muñoz, F. X.; Banks, C. E.; Compton, R. G. Electroanalysis 2007, 19, 1973-1986. 
(15) Wehmeyer, K. R.; Wightman, R. M. J. Electroanal. Chem. Interfacial Electrochem. 1985, 196, 417-421.

(16) Wipf, D. O.; Michael, A. C.; Wightman, R. M. J. Electroanal. Chem. Interfacial Electrochem. 1989, 269, 15-25.

(17) Cinková, K.; Clark, M.; Sokolov, S. V.; Batchelor-McAuley, C.; Švorc, L.; Compton, R. G. Electroanalysis 2016.

(18) Ellison, J.; Batchelor-McAuley, C.; Tschulik, K.; Compton, R. G. Sens. Actuators, B 2014, 200, 47-52.

(19) Batchelor-McAuley, C.; Ellison, J.; Tschulik, K.; Hurst, P. L.; Boldt, R.; Compton, R. G. The Analyst 2015, 140, 5048-5054.

(20) Szabo, A.; Cope, D. K.; Tallman, D. E.; Kovach, P. M.; Wightman, R. M. J. Electroanal. Chem. 1987, 217, 417-423.

(21) Aoki, K.; Honda, K.; Tokuda, K.; Matsuda, H. J. Electroanal. Chem. 1985, 182, 267-279.

(22) Miao, W.; Ding, Z.; Bard, A. J. J. Phys. Chem. B 2002, 106, 1392-1398.

(23) Michelet, F.; Gueguen, R.; Leroy, P.; Wellman, M.; Nicolas, A.; Siest, G. Clin. Chem. 1995, 41, 1509-1517.

(24) Zappacosta, B.; Manni, A.; Persichilli, S.; Boari, A.; Scribano, D.; Minucci, A.; Raffaelli, L.; Giardina, B.; De Sole, P. Clin. Biochem. 2007, 40, 661-665.

(25) Franco, R.; Schoneveld, O. J.; Pappa, A.; Panayiotidis, M. I. Arch. Physiol. Biochem. 2007, 113, 234-258.

(26) Ballatori, N.; Krance, S. M.; Notenboom, S.; Shi, S.; Tieu, K.; Hammond, C. L. Biol. Chem. 2009, 390, 191-214.

(27) Lawrence, N.; Davis, J.; Compton, R. G. Talanta 2001, 53, 1089-1094.

(28) Szajewski, R. P.; Whitesides, G. M. J. Am. Chem. Soc. 1980, 102, 2011-2026.

(29) Ngamchuea, K.; Batchelor-McAuley, C.; Compton, R. G. Chem. - Eur. J. 2016, 22, 15937-15944.

(30) Ward, K. R.; Lawrence, N. S.; Hartshorne, R. S.; Compton, R. G. J. Phys. Chem. C 2011, 115, 11204-11215.

(31) Jiao, X.; Lin, C.; Young, N. P.; Batchelor-McAuley, C.; Compton, R. G. J. Phys. Chem. C 2016, 120, 13148-13158.

(32) Compton, R. G.; Laborda, E.; Ward, K. R. Understanding voltammetry: simulation of electrode processes; Imperial College Press, 2013.

(33) Xiong, L.; Aldous, L.; Henstridge, M. C.; Compton, R. G. Anal. Methods 2012, 4, 371-376.

(34) Sun, P.; Mirkin, M. V. Anal. Chem. 2006, 78, 6526-6534.

(35) Nekrassova, O.; Lawrence, N. S.; Compton, R. G. Electroanalysis 2003, 15, 15011505.

(36) Bard, A. J.; Faulkner, L. R. Electrochemical Methods: Fundamentals and Applications; Wiley, 2000. 


\section{For TOC Only}

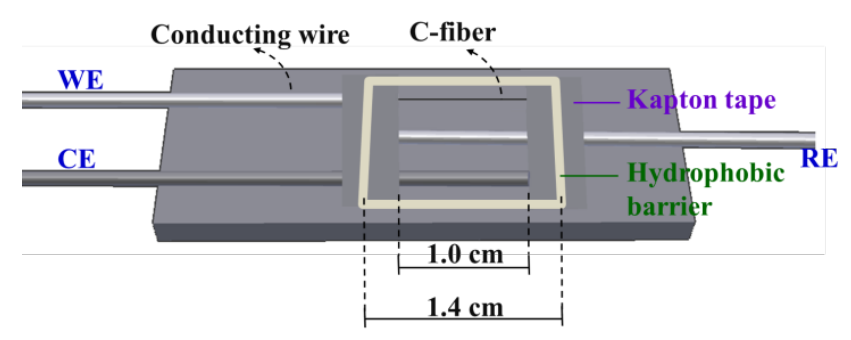

